\title{
Structure of the Azzarelli-Collas representation for the scattering amplitude and generalization to the Rice representation and the Euler-Pochhammer representation
}

\author{
Alfred C. T. Wu \\ Department of Physics, University of Michigan, Ann Arbor, Michigan 48109 \\ (Received 11 April 1977)
}

The Azzarelli-Collas integral representation for the scattering amplitude is extended to the Rice representation. The latter representation in turn is viewed as an example of the Euler-Pochhammer representation.

\section{INTRODUCTION}

Recently, Azzarelli and Collas have derived a new integral representation for the scattering amplitude. ${ }^{1}$ The representation originated from their study of Coulomb scattering in the parabolic coordinate system where the Schrödinger equation is separable and the wavefunctions are expressible as a superposition of products of Whittaker functions. A general derivation is obtained ${ }^{1}$ by recasting, in the partial wave expansion, the Legendre function in terms of the Bateman function. ${ }^{2}$ It appears that the partial wave amplitude smeared over by the Bateman function is a much smoother function in the new variable. Usual Regge poles in the angular momentum plane may now appear however as zeroes in this new formalism. ${ }^{1}$

We study here the structure of the Azzarelli-Collas integral representation and the generalization the reof.

The Azzarelli-Collas representation may be viewed as a special Mellin transform. It so happens that the same relationship exists between the Bateman function and the Legendre function. ${ }^{3}$ This last fact permits the kernel in the integral representation (or the expansion coefficients in the power series expansion via a Sommerfeld-Watson transformation) to be simply related to the partial wave amplitude.

In their derivation of the integral representation, Azzarelli-Collas used an identity between the Legendre function and the Bateman function, and cited the reference to Rice. ${ }^{3}$ Rice has actually studied a generalization of the Bateman function, which we shall refer to as the Rice function. The use of the Rice function in place of the Bateman function gives an immediate generalization of the Azzarelli-Collas representation to a new representation which we shall refer to as the Rice representation.

The Rice representation may be viewed further as an example of the Euler-Pochhammer representation. ${ }^{4}$ Previously, other examples of the generalized EulerPochhammer representations have been found in the study of Feynman amplitudes, ${ }^{5}$ Veneziano amplitudes, ${ }^{6}$ angular momentum recoupling coefficients, ${ }^{7}$ and $\mathrm{SU}(N)$ basis functions. ${ }^{8}$

For the sake of readability, we give briefly in Sec. II a derivation of the Azzarelli-Collas representation from first principles. In Sec. III, we emphasize the
Euler-Pochhammer aspect of the representation. In Sec. IV, we generalize the Azzarelli-Collas representation to the Rice representation. The EulerPochhammer structure is manifest.

Although the Azzarelli-Collas representation owes its origin to the analysis of the Coulomb scattering in the parabolic coordinates, the heuristic argument presented in Ref. 1 regarding the asymptotic behavior of the Whittaker functions does not seem to provide a deeper insight to the raison d'être of the integral representation. One possible interpretation from the group-theoretic point of view would be as follows. The spherical functions pertaining to the rank-one $O(3)$ group and labelled by the angular momentum $l$ (beside the third component label $m$ ) belong to the family of the Gauss hypergeometric function [such as $P_{1}(z)=$ ${ }_{2} F_{1}(-l, l+1 ; 1 ;(1-z) / 2]$. The case of the Coulomb scattering admits, as is well known, the group $O(4)$, which is of rank two. Therefore, it is quite conceivable to arrange, at least, for some of the representation functions to correspond to the higher-hierarchy generalized hypergeometric functions [such as ${ }_{3} F_{2}(-l, l+1$, $\nu ; 1, p ; z)$ whence the Bateman and the Rice functions] to accommodate the extra label. In this spirit, it would be interesting to view the generalized Euler-Pochhammer integral representation as natural for the cases of underlying symmetry groups of higher ranks.

\section{AZZARELLI-COLLAS INTEGRAL REPRESENTATION AS A MELLIN TRANSFORM}

The Azzarelli-Collas integral representation for the scattering amplitude can be derived from first principles as follows.

Step 1: Expand the scattering amplitude $A(s, z)$ into partial wave amplitudes

$$
A(s, z)=\sum_{l=0}^{\infty}(2 l+1) a_{l}(s) P_{l}(z)
$$

Step 2: Recognize that the Legendre function $P_{t}$ is a special case of the Gauss hypergeometric function ${ }_{2} F_{1}$,

$$
P_{l}(z)={ }_{2} F_{1}\left(-l, l+1 ; 1 ; \frac{1}{2}(1-z)\right)
$$

and that the Bateman function $F_{t}$ is a special case of the ${ }_{3} F_{2}$ function,

$$
F_{l}(-2 \nu-1)={ }_{3} F_{2}(-l, l+1, \nu ; 1,1 ; 1) .
$$


Step 3: Recall that there is a Euler-Pochhammer integral relation between ${ }_{A+1} F_{B+1}$ and ${ }_{A} F_{B}{ }^{4}$

$$
\begin{aligned}
& { }_{A+1} F_{B+1}((a), c ;(b), d ; w) \\
& \quad=\frac{\Gamma(d)}{\Gamma(c) \Gamma(d-c)} \int_{0}^{1} d t t^{r-1}(1-t)^{d-c-1} \quad{ }_{A} F_{B}((a) ;(b) ; w t),
\end{aligned}
$$

where $(a)$ and $(b)$ are shorthand notations for the two sets of parameters $a_{1}, \ldots, a_{A}$ and $b_{1}, \ldots, b_{B}$ respectively. Applying (4) to (3) and (2) for the case $A=2$ and $B=1$ gives the Euler-Pochhammer representation for the Bateman function, namely

$$
\begin{aligned}
F_{i}(-2 \nu-1) \\
=-\frac{\sin \pi \nu}{\pi} \int_{0}^{1} d t t^{-\nu-1}(1-t)_{2}^{\nu} F_{1}(-l, l+1 ; 1 ; t) \\
=-\frac{\sin \pi \nu}{\pi} \int_{0}^{1} d t t^{-\nu-1}(1-t)^{\nu} P_{l}(1-2 t) \\
=-\frac{\sin \pi \nu}{\pi} \int_{0}^{\infty} d \tau \tau^{-\nu-1}(1+\tau)^{-1} P\left(\frac{1-\tau}{1+\tau}\right),
\end{aligned}
$$

where $t=\tau /(1+\tau)$ in going from (5) to (6). Equation (5) is complementary to the contour representation Eq. (B1) quoted in Ref. 1.

Step 4: Equation (6) may be viewed as the Mellin transform of $(1+\tau)^{-1} P((1-\tau) /(1+\tau))$. The inverse Mellin transform gives

$$
\begin{aligned}
& (1+\tau)^{-1} P\left(\frac{1-\tau}{1+\tau}\right) \\
& \quad=\frac{1}{2 \pi i} \int_{\sigma-i \infty}^{\sigma+i \infty} d \nu \tau\left(\frac{-\pi F_{l}(-2 \nu-1)}{\sin \pi \nu}\right),
\end{aligned}
$$

where $(1-\tau) /(1+\tau)=z=\cos \theta$. This is the Eq. (3) quoted in Ref. 1 and attributed to Rice. ${ }^{3}$

Step 5: Substituting (7) into (1) gives the AzzarelliCollas representation

$$
\begin{aligned}
& (1+\tau)^{-1} A\left(s, \frac{1-\tau}{1+\tau}\right) \\
& \quad=\frac{1}{2 \pi i} \int_{\sigma=i \infty}^{\sigma+i \infty} d \nu \tau^{\nu}\left(\frac{-\pi b(s, \nu)}{\sin \pi \nu}\right) \\
& \quad=\sum_{\nu=0}^{\infty} b(s, \nu)(-\tau)^{\nu}, \\
& \frac{-\pi b(s, \nu)}{\sin \pi \nu}=\int_{0}^{\infty} d \tau \tau^{-\nu-1}(1+\tau)^{-1} A\left(s, \frac{1-\tau}{1+\tau}\right) .
\end{aligned}
$$

Equations (8a) and (8c) may be viewed simply as the Mellin transforms between $(1+\tau)^{-1} A(s,(1-\tau) /(1+\tau))$ and $-\pi b(s, \nu) / \sin \pi \nu$. This $b(s, \nu)$ is simply related to the partial wave amplitudes $a_{l}(s)$ in the following sense. ${ }^{1}$ As the folded sum of $a_{t}(s)$ with the Legendre functions $P_{l}(z)$ gives $A(s, z)$, the folded sum of $a_{l}(s)$ with the Bateman function $F_{l}(-2 v-1)$ gives $b(s, v)$,

$$
b(s, \nu)=\sum_{l=0}^{\infty}(2 l+1) a_{l}(s) F_{l}(-2 v-1) .
$$

Equations ( $8 b)$ and ( $8 a)$ are related by a Sommerfeld-
Watson transformation. A convenient way to remember the structure of the Azzarelli-Collas representation $(8)$ is that setting $b(s, \nu) \equiv 1$ formally in $(8 \mathrm{~b})$ gives $A=1$, and Eqs. (8a) and (8c) then merely confirm the fact that $(1+\tau)^{-1}$ and $\pi / \sin \pi \nu$ are a Mellin-transform pair.

\section{EXTENSION TO THE EULER-POCHHAMMER REPRESENTATION}

In terms of the variable $t=\tau /(1+\tau)$, Eq. (8c) reads

$$
\begin{aligned}
b(s, \nu)= & \frac{\Gamma(1)}{\Gamma(-\nu) \Gamma(1+\nu)} \\
& \times \int_{0}^{1} d t t^{-\nu-1}(1-t)^{\nu} A(s, 1-2 t) .
\end{aligned}
$$

This is a special case of the Euler-Pochhammer representation

$$
\begin{aligned}
g(z ; c, d)= & \frac{\Gamma(d)}{\Gamma(c) \Gamma(c-d)} \\
& \times \int_{0}^{1} d t t^{-1}(1-t)^{d-c-1} f(t ; z)
\end{aligned}
$$

evaluated at $c=-\nu, d=1$.

Examples of the representation (11) and the generalization the reof to multiple integrals have been cited by the statements in the Introduction. It is expected that such a generalized Euler-Pochhammer integral representation would occur, for example, in the multiple scattering.

\section{THE RICE REPRESENTATION}

In place of the Bateman function (3), the Rice function $^{3}$ is given by

$$
R_{1}(\nu, p, z) \equiv{ }_{3} F_{2}(-l, l+1, \nu ; 1, p ; z) .
$$

The Bateman function is recovered in the limit $p=1$ and $z=1$,

$$
R_{l}(\nu ; 1 ; 1)=F_{l}(-2 \nu-1) .
$$

The remarkable feature of Rice's analysis is that the integral relation (4) between ${ }_{3} F_{2}$ and ${ }_{2} F_{1}$ is actually invertible, namely

$$
\begin{aligned}
{ }_{2} F_{1}\left(a_{1}, a_{2} ; b_{1} ; z\right) \\
=\frac{\Gamma\left(a_{3}\right)}{\Gamma\left(b_{2}\right) \Gamma\left(a_{3}-b_{2}\right)} \int_{0}^{1} d u u^{b_{2}-1}(1-u)^{a_{3}-b_{2}-1} \\
\quad \times_{3} F_{2}\left(a_{1}, a_{2}, a_{3} ; b_{1}, b_{2} ; z u\right) .
\end{aligned}
$$

This implies that from the relations (1), (2), and (14), we have

$$
\begin{aligned}
& A(s, 1-2 w) \\
& \quad=\sum_{l}(2 l+1) a_{l}(s) P_{l}(1-2 w) \\
& \quad=\frac{\Gamma(\nu)}{\Gamma(p) \Gamma(\nu-p)} \int_{0}^{1} d u u^{p-1}(1-u)^{\nu-p-1} r(s, \nu, p ; w u), \\
& r(s, \nu, p ; y) \\
& \quad=\sum_{l}(2 l+1) a_{l}(s) R_{l}(\nu, p, y)
\end{aligned}
$$




$$
=\frac{\Gamma(p)}{\Gamma(\nu) \Gamma(\nu-p)} \int_{0}^{1} d t t^{\nu-1}(1-t)^{p-\nu-1} A(s, 1-2 y t) .
$$

Equation (16) is the generalization of (9) while Eq. (17) is that of (10). On the other hand, unlike the AzzarelliCollas reprentation (8a) which is merely a Mellin transform, Eq. (15) is also in the form of the EulerPochhammer representation.

${ }^{1}$ T. Azzarelli and P. Collas, Phys, Rev. D 12, 3237 (1975).
${ }^{2}$ Higher Transcendental Functions (Bateman Manuscript Project), edited by A. Erdelyi, et al. (McGraw-Hill, New York, 1953), Vol. 1, esp. p. 192.

${ }^{3}$ S.O. Rice, Duke Math. J. 6, 108 (1939).

${ }^{4}$ See, e.g. , J.J. Slater, Generalized Hypergeometric Functions (Cambridge U.P., Cambridge, 1966)

${ }^{5}$ A. C. T. Wu, Phys. Rev. D 9, 370 (1974).

${ }^{6}$ A. C. T. Wu, J. Math. Phys, 12, 2035 (1971).

${ }^{7}$ A.C.T. Wu, J. Math. Phys. 14, 1222 (1973).

${ }^{8}$ A.C.T. Wu, J. Math. Phys. 12, 437 (1971); C. S. Huang and A.C.T. Wu, J. Math. Phys. 14, 263 (1973). 